\title{
Dosimetric evaluation of Point A and volume-based high-dose-rate plans: a single institution study on adaptive brachytherapy planning for cervical cancer
}

\author{
Arun George Paul, MD, PhD',2, Adrian Nalichowski, PhD³ , Judith Abrams, PhD²,3, Peter Paximadis, MD², \\ Ling Zhuang, PhD², Steven Miller MD² \\ 'Detroit Medical Center, ${ }^{2}$ Wayne State University School of Medicine, ${ }^{3}$ Barbara Ann Karmanos Cancer Institute, Detroit, MI, USA
}

\begin{abstract}
Purpose: External beam radiation therapy (EBRT) and brachytherapy (BT) with concurrent cisplatin is the standard of care for locally advanced cervical cancer. The applicability of image-guided adaptive volume-based high-dose-rate (HDR) intracavitary brachytherapy planning is an active area of investigation. In this study, we examined whether volume-based HDR-BT $\left(\mathrm{HDR}_{\mathrm{VOL}}\right)$ plans leads to more conformal plans compared to Point $\mathrm{A}\left(\mathrm{HDR}_{\text {Point } \mathrm{A}}\right)$-based plans.

Material and methods: Two hundred and forty $\mathrm{HDR}_{\text {Point }}$ plans from 48 cervical cancer patients treated with chemoradiotherapy were retrospectively collected. Point A plans were renormalized with respect to the high-risk clinical target volume (HR-CTV) for the $\mathrm{HDR}_{\mathrm{VOL}}$ plans. The doses to organs at risk (OAR; rectum, sigmoid, and bladder), and HR-CTV and the conformal index were compared between $\mathrm{HDR}_{\text {PointA }}$ and $\mathrm{HDR}_{\mathrm{VOL}}$ plans.

Results: $\mathrm{HDR}_{\mathrm{VOL}}$ plans resulted in a 6-12\% reduction in the total dose (EBRT + HDR-BT) to $0.1 \mathrm{cc}, 1.0 \mathrm{cc}$, and $2.0 \mathrm{cc}$ of the OAR as well as an $8-37 \%$ reduction in the dose to 2 cc of OAR per HDR-BT fraction compared to HDR PointA $_{\text {plans. }}$ Differences in the conformal indexes between the two groups of plans showed an $18-31 \%$ relative increase per HDR-BT fraction for $\mathrm{HDR}_{\mathrm{VOL}}$ plans. The $\mathrm{D}_{90}$ of the HR-CTV was reduced by $11 \%$ by $\mathrm{HDR}_{\mathrm{VOL}}$ planning and had a median dose of 86 Gy.

Conclusions: Our study reports the relative improvement in OAR doses per HDR-BT fraction by HDR $\mathrm{VOL}_{\text {planning }}$ compared to $\mathrm{HDR}_{\text {PointA }}$ planning and demonstrates the dosimetric advantages of volume-based HDR-BT planning in creating more conformal plans.
\end{abstract}

J Contemp Brachytherapy 2018; 10, 3: 202-210 DOI: https://doi.org/10.5114/jcb.2018.76782

Key words: brachytherapy, cervical cancer, HDR, image-guided, Point A, radiation-planning.

\section{Purpose}

Radiation therapy (RT) with concurrent weekly cisplatin consisting of external beam radiation therapy (EBRT) and brachytherapy (BT) is the standard of care for locally advanced cervical cancers [1,2]. Local control rates greater than $80 \%$ requires a total biological equivalent dose in $2 \mathrm{~Gy}$ per fraction $\left(\mathrm{EQD}_{2}\right)$ of $80-90$ Gy or more to the high-risk clinical target volume (HR-CTV) [3,4,5,6]. A BT-based approach by low-dose-rate or high-dose-rate (HDR) is most commonly used to achieve a total $\mathrm{EQD}_{2}(\mathrm{EBRT}+\mathrm{BT})$ dose of 80-90 Gy to the HR-CTV [1,2]. The sharp dose fall-off allows for high $\mathrm{EQD}_{2}$ HR-CTV doses without compromising organs at risk (OAR) doses [1,2,7,8]. Lately, HDR-BT has become more common with the recent report by the quality research in radiation oncology study showing its application in $60-70 \%$ of the surveyed facilities $[9,10]$.
HDR-BT for cervical cancer uses the Manchester Point A-based system and has been an effective modality for delivering high-dose radiation to the HR-CTV [7,8,11,12]. The strength of this system is its simplicity in implementation and its reproducibility with respect to the bony anatomy when assessed by radiographs. The main disadvantage of Point A system is related to dose delivery uncertainties due to inter-fraction and intra-fraction geometric disparities in applicator positions, and anatomical variations in rectal and bladder fillings resulting in non-reproducible high- and low-dose areas within the OAR $[13,14,15]$. Clinically, this could translate into underdosing and over-dosing of OAR. With the advent of computed tomography/magnetic resonance imaging (CT/MRI) scans and CT/MRI compatible HDR applicators, the accuracy of HR-CTV and OAR dose assessment significantly
Address for correspondence: Steven Miller, MD, Assistant Professor, Wayne State University School of Medicine, 4100 John R, Detroit, MI, USA, phone: +1 313-576-9545, fax: +1 313-576-9625,

e-mail: srmiller@med.wayne.edu
Received: 08.01 .2018

Accepted: 18.04 .2018

Published: 30.06 .2018 
improved since the target and OAR were contoured and used for planning as demonstrated by multiple studies $[16,17,18,19]$. In recent years, several studies have indicated the benefits of volume-based HDR-BT planning $[19,20,21,22,23,24,25]$. Volume-based HDR-BT planning creates more conformal treatment plans after careful evaluation of the dose cloud within the context of HR-CTV and OAR contoured from either CT or MRI obtained per HDR-BT fraction $[19,20,21]$. In this study, we examined the dosimetric outcomes of volume-based HDR-BT $\left(\mathrm{HDR}_{\mathrm{VOL}}\right)$ planning for locally advanced cervical cancers and quantifies the relative difference in OAR doses per HDR-BT fraction between conventional Point A and volume-based HDR-BT planning.

\section{Material and methods}

\section{Study population}

From January 2008 to July 2015, 124 patient charts with locally advanced cervical cancer treated with concurrent chemotherapy (cisplatin) and RT (EBRT + HDR-BT) were retrospectively screened for the study after approval from the institutional review board. The inclusion criteria used for the screening were: a) CT scan for each HDR-BT fraction ( $f x$ ); b) five HDR-BT fractions (fxs) using tandem and ring (T\&R) or tandem and ovoids (T\&O); c) completion of planned radiation treatment; d) absence of metastatic disease; e) availability of electronic HDR-BT planning records. All patients were staged according to the International Federation of Gynecology and Obstetrics (FIGO) classification [26].

\section{Radiation techniques}

RT comprised of whole pelvic RT of 45 Gy (1.8 Gy per fraction with five fractions per week) inter-digitated with HDR-BT. Para-aortic chains were treated for patients with either pelvic lymph node or para-aortic node involvement. EBRT was delivered by either intensity modulated RT (IMRT) or three-dimensional RT (3DRT) after delineating the target volumes and OAR on CT scans. MRI and positron emission tomography (PET) scans were fused with the CT images whenever available. Gross nodal involvement received additional boost RT. HDR-BT consisted of twice weekly treatments. CT scans were obtained on a large bore Somatom Sensation CT simulator (Siemens, Malvern, PA, USA) for each HDR-BT fraction after the insertion of CT compatible applicators tandem and ring (T\&R) and tandem and ovoid (T\&O) with sufficient vaginal packing for ensuring appropriate placements and planning. $60 \mathrm{cc}$ of normal saline was injected into the bladder by Foley catheter before obtaining the planning CT scan and was left in situ for each HDR-BT fraction. The rectum, sigmoid, bladder, and HR-CTV were contoured by a radiation oncologist on 3-5 $\mathrm{mm}$ axial CT slices on the CT scan for the respective HDR-BT $f x$ as per the American Brachytherapy Society (ABS) guidelines [1], and was used for HDR-BT planning using Oncentra HDR planning software (Elekta, Stockholm, Sweden). MRI was used for delineating HR-CTV by fusing with the CT image, whenever a second MRI was obtained during EBRT to assess tumor response as previously described [27]. The prescription points used were either Point A or modified Point A as previously described $[2,28,29,30]$. Point A was defined as the point $2 \mathrm{~cm}$ superior to the external os along the tandem, and $2 \mathrm{~cm}$ perpendicular from the intrauterine tandem $[2,28,29,30]$. Modified Point A plans were chosen when Point A planning resulted in excessive OAR doses for patients with smaller HR-CTV volumes. Modified Point A-based plans were created by changing the prescription point from Point A by altering the lateral distance from the tandem from $2 \mathrm{~cm}$ to anywhere between $1.6 \mathrm{~cm}$ and $1.9 \mathrm{~cm}$.

\section{High-risk clinical target volume-based high-dose-rate brachytherapy planning protocol}

Two hundred forty $\mathrm{HDR}_{\text {PointA }}$ plans from 48 patients with five HDR-BT fractions per patient were reviewed and re-planned with respect to HR-CTV volume for $\mathrm{HDR}_{\mathrm{VOL}}$ plans. Each plan was re-normalized by scaling the source dwell times and re-optimized by graphically adjusting the isodose lines with objective of lowering OAR doses and at the same time, achieving at a minimum either the $D_{95}\left(D_{95 P R x}\right)$ or the $D_{90}$ of the HR-CTV $\left(D_{90 \text { PRx }}\right)$ to be the prescription dose. If the initial $D_{95}\left(D_{95 \text { INITIAL }}\right)$ or the initial $\mathrm{D}_{90}\left(\mathrm{D}_{\text {90INITIAL }}\right)$ of the HR-CTV were lower than the prescription dose due to the proximity of OAR, an attempt was made to re-optimize by graphically adjusting the isodose lines to achieve maximum OAR sparing followed by re-normalizing to either the $\mathrm{D}_{\text {95INITIAL }}$ or $\mathrm{D}_{90 \text { INITIAL. }}$ If the afore mentioned requirements were not met, the respective plans were rejected and the initial Point A plan (Point A) was accepted for analysis. The $\mathrm{D}_{0.1(\mathrm{HDR})}$ (dose to $0.1 \mathrm{cc}$; data not shown), $\mathrm{D}_{1.0(\mathrm{HDR})}$ (dose to $1.0 \mathrm{cc}$; data not shown), and $\mathrm{D}_{2.0(\mathrm{HDR})}$ (dose to $2.0 \mathrm{cc}$ ) of rectum, sigmoid, and bladder and the $\mathrm{D}_{90 \text { (HDR) }}$ (dose to $90 \%$ of HR-CTV; data not shown), $\mathrm{V}_{150 \text { (HDR) }}$ (percentage of HR-CTV receiving $150 \%$ of the prescription dose), and $\mathrm{V}_{200 \text { (HDR) }}$ (percentage of HR-CTV receiving 200\% of the prescription dose) were collected for both $\mathrm{HDR}_{\text {PointA }}$ and $\mathrm{HDR}_{\mathrm{VOL}}$ plans per HDR-BT fraction. The total EQD 2 doses $\mathrm{D}_{0.1(\mathrm{EBRT}+\mathrm{HDR})}, \mathrm{D}_{1.0(\mathrm{EBRT}+\mathrm{HDR})}$, and $\mathrm{D}_{2.0(\mathrm{EBRT}+\mathrm{HDR})}$ of the rectum, sigmoid, and bladder, and the $\mathrm{D}_{90(\mathrm{EBRT}+\mathrm{HDR})}$ of the HR-CTV from the combination of EBRT and HDR-BT were calculated for each patient using ABS worksheet ("HDR Radiobiologic Dose Equivalent Worksheets \#2", https:/ / www.americanbrachytherapy.org/guidelines/) for dose calculations. Conformal index for each HDR-BT plan was calculated as previously described [21]. Conformal index $=\left(\mathrm{CTV}_{\text {ref }} / \mathrm{V}_{\mathrm{CTV}}\right) \times\left(\mathrm{CTV}_{\text {ref }} / \mathrm{V}_{\text {ref }}\right) . \mathrm{CTV}_{\text {ref }}$ is defined as the volume of the HR-CTV receiving $\geq 100 \%$ dose. $V_{\text {CTV }}$ is defined as the volume of the HR-CTV. $\mathrm{V}_{\text {ref }}$ is defined as the volume receiving a dose $\geq 100 \%$ dose [21].

\section{Statistical analysis}

Estimates of proportions are reported with $95 \%$ confidence intervals. Estimates of continuous quantities are reported as medians and interquartile ranges (IQR) because of the asymmetry of the frequency distributions. Relative differences of various dosimetric parameters $\left(\mathrm{D}_{0.1(\mathrm{EBRT}+\mathrm{HDR})}, \mathrm{D}_{1.0(\mathrm{EBRT}+\mathrm{HDR})}, \mathrm{D}_{2.0(\mathrm{EBRT}+\mathrm{HDR})}\right.$, and $\left.\mathrm{D}_{2.0(\mathrm{HDR})}\right)$ 
of the rectum, sigmoid, and bladder $\left(\mathrm{D}_{90(\mathrm{EBRT}+\mathrm{HDR})}, \mathrm{V}_{150(\mathrm{HDR})}\right.$ and $\mathrm{V}_{200(\mathrm{HDR})}$ ) of the HR-CTV, and the conformal indexes were calculated as 100 times the difference between $\mathrm{HDR}_{\text {PointA }}$ and $\mathrm{HDR}_{\mathrm{VOL}}$ divided by $\mathrm{HDR}_{\mathrm{VOL}}$.

\section{Results}

\section{Population and radiation therapy characteristics}

Forty-eight patients met the inclusion criteria of the study with median follow-up of 13 months. Sixty-four percent of the patients had locally advanced cancer ( $\geq$ IIA), whereas $11 \%$ and $25 \%$ of the patients had IB1 and IB2 disease, respectively. Vaginal involvement was seen in $10 \%$ of the patients with either proximal (IIA2, $6 \%$ ) or distal (IIIA, $4 \%$ ) vaginal involvement. Parametrial/adnexal involvement was present in $29 \%$ (IIB) of the patients. Twenty-three percent of the patients had stage IIIB disease at diagnosis with either pelvic sidewall involvement or hydronephrosis or non-functioning kidney. Patients characteristics are presented in Table 1.

Sixty-seven percent of the patients received fractionated parametrial or sidewall boost RT by 3 DRT with doses ranging from 1.8-9.0 Gy. Fifty-two percent of the patients received fractionated nodal boost RT with doses ranging from 3.6-19.6 Gy. HDR-BT treatments were inter-digitated with EBRT with median total RT (EBRT + HDR-BT) treatment time of 61 days and median total HDR-BT time of 17 days (Tables 2 and 3). RT characteristics are presented in Table $2.94 \%$ and $2 \%$ of the patients

Table 1. Population characteristics

\begin{tabular}{lc}
\hline Parameters & $N=48$ \\
\hline Age at diagnosis (years), median (IQR) & $49(40,59)$ \\
\hline Race & $36(75 \%)$ \\
\hline AA & $12(25 \%)$ \\
\hline Stage & \\
\hline IB1 & $5(11 \%)$ \\
\hline IIA2 & $12(25 \%)$ \\
\hline IIB & $3(6 \%)$ \\
\hline IIIA & $14(29 \%)$ \\
\hline IIIB & $2(4 \%)$ \\
\hline Pathology & $11(23 \%)$ \\
\hline Squamous cell carcinoma & $1(2 \%)$ \\
\hline Adenocarcinoma & $42(88 \%)$ \\
\hline Others & $2(4 \%)$ \\
\hline Follow-up (months), median (IQR) & $4(8 \%)$ \\
\hline
\end{tabular}

Table 2. Radiation therapy characteristics

\begin{tabular}{lc}
\hline Parameters & $N=48$ \\
\hline EBRT dose & $48(100 \%)$ \\
\hline 45 Gy & \\
\hline EBRT modality & $24(50 \%)$ \\
\hline IMRT & $24(50 \%)$ \\
\hline Sidewall boost & $32(67 \%)$ \\
\hline Mod patients & $32(67 \%)$ \\
\hline 3DRT & $1(2 \%)$ \\
\hline Dose (Gy) & $25(52 \%)$ \\
\hline 5.8 & $6(13 \%)$ \\
\hline 9
\end{tabular}

\section{Nodal boost}

\begin{tabular}{ll}
\hline \# of patients & 25 (52\%) \\
\hline
\end{tabular}

Modality

\begin{tabular}{lc}
\hline 3DRT & $8(17 \%)$ \\
\hline IMRT & $14(29 \%)$ \\
\hline $3 D R T$ \& IMRT & $3(6 \%)$
\end{tabular}

Dose (Gy)

\begin{tabular}{ll}
\hline 3.6 & $2(4.2 \%)$ \\
\hline 4 & $1(2.1 \%)$ \\
\hline
\end{tabular}

$6 \quad 4(8.3 \%)$

$7.5 \quad 1(2.1 \%)$

$8 \quad 2(4.2 \%)$

$93(6.3 \%)$

$10 \quad 1(2.1 \%)$

$10.8 \quad 5(10.4 \%)$

$12.6 \quad 1(2.1 \%)$

$14 \quad 2(4.2 \%)$

$14.4 \quad 1(2.1 \%)$

$16 \quad 1(2.1 \%)$

19.6

$1(2.1 \%)$

\begin{tabular}{ll} 
Duration (days), median (IQR) & \\
\hline RT (EBRT + brachytherapy) & $61(53,68)$
\end{tabular}

$N$ - total number of patients, EBRT - external beam radiation therapy, $3 D$ three dimensional, IMRT - intensively modulated radiation therapy 
used T\&R and T\&O applicators exclusively, respectively. $48 \%$ of the patients were treated with ' $5.5 \mathrm{~Gy} \times 5^{\prime}$ HDR-BT fractionation regimen, and $52 \%$ of the patients received other commonly used fractionation schemes. Median volumes of HR-CTV, rectum, sigmoid, and bladder from the $240 \mathrm{HDR}_{\text {PointA }}$ plans reviewed were $23 \mathrm{cc}, 52 \mathrm{cc}$, $44 \mathrm{cc}$, and $100 \mathrm{cc}$, respectively. Treatment characteristics are shown in Table 3. Grade 3-4 gastrointestinal (GI) and genitourinary (GU) toxicities were observed in $17 \%$ and $15 \%$ of the patients, respectively, with a median follow-up of 13 months, and five $(10 \%)$ of the 48 patients were lost to follow-up (Tables 1 and 4).

\section{High-dose-rate brachytherapy planning features}

Seventy-one percent of the 48 patients had Point A-based prescriptions for all five HDR-BT fractions (Table 3). Twenty-nine percent of the 48 patients used modified point A-based prescriptions as part of the overall brachytherapy fractionation regimen (Table 3). Among the 240 plans reviewed from 48 patients (5 HDR-BT plans per patient), $80 \%$ (192/240 plans) were Point A plans and $20 \%$ (48/240 plans) were modified Point A plans. Modified Point A prescription points used were $1.9 \mathrm{~cm} \mathrm{(2/240}$ plans, $0.8 \%), 1.8 \mathrm{~cm}(27 / 240$ plans, $11.3 \%), 1.7 \mathrm{~cm} \mathrm{(14/240}$ plans, $5.8 \%)$, and $1.6 \mathrm{~cm}$ (5/240 plans, $2.1 \%)$. Planning strategies for $\mathrm{HDR}_{\mathrm{VOL}}$ plans consisted of re-normalizing the prescription dose to $\mathrm{D}_{95 \mathrm{PRx}}(176 / 240$ plans, $73.3 \%)$, $\mathrm{D}_{\text {90PRx }}(31 / 240$ plans, $13 \%), \mathrm{D}_{\text {95INITIAL }}$ (2/240 plans, $\left.0.8 \%\right)$, and $\mathrm{D}_{\text {90INITIAL }}(2 / 240$ plans, $0.8 \%)$. Volume-based planning could not improve OAR doses without compromising initial HR-CTV doses for $12.1 \%$ (29/240 plans) of the plans reviewed and thus the initial plan (Point A) was used for analysis.

\section{Point A and volume-based high-dose-rate planning comparisons}

The EQD 2 doses $\mathrm{D}_{0.1(\mathrm{EBRT}+\mathrm{HDR})}, \mathrm{D}_{1.0(\mathrm{EBRT}+\mathrm{HDR}) \text {, and }}$ $\mathrm{D}_{2.0 \text { (EBRT+HDR) }}$ was decreased by $\mathrm{HDR}_{\mathrm{VOL}}$ planning with a relative difference of $8-10 \%$ for rectum, $6-9 \%$ for sigmoid, and $10-12 \%$ for bladder compared to $\mathrm{HDR}_{\text {PointA }}$ plans (Table 5). The median $\mathrm{D}_{0.1(\mathrm{EBRT}+\mathrm{HDR})}, \mathrm{D}_{1.0(\mathrm{EBRT}+\mathrm{HDR})^{\prime}}$ and $\mathrm{D}_{2.0(\mathrm{EBRT}+\mathrm{HDR})}$ for $\mathrm{HDR}_{\text {PointA }}$ and $\mathrm{HDR}_{\mathrm{VOL}}$ plans were $73 \mathrm{~Gy}$ and $63 \mathrm{~Gy}, 66 \mathrm{~Gy}$ and $58 \mathrm{~Gy}, 63 \mathrm{~Gy}$ and $56 \mathrm{~Gy}$ for rectum, $76 \mathrm{~Gy}$ and $70 \mathrm{~Gy}, 68 \mathrm{~Gy}$ and $62 \mathrm{~Gy}, 65 \mathrm{~Gy}$ and 59 Gy for sigmoid, and 91 Gy and 78 Gy, 79 Gy and $68 \mathrm{~Gy}$, and $74 \mathrm{~Gy}$ and $66 \mathrm{~Gy}$ for bladder, respectively (Table 5). $\mathrm{HDR}_{\mathrm{VOL}}$-based planning decreased the $\mathrm{D}_{2.0(\mathrm{HDR})}$ per HDR-BT $\mathrm{fx}$ for all the plans with a relative difference of $19-37 \%$ for rectum, $10-26 \%$ for sigmoid, and $8-23 \%$ for bladder compared to $\mathrm{HDR}_{\text {PointA }}$ plans for fractions 1-5 (Table 5). The median rectal $D_{2.0(\mathrm{HDR})}$ for fractions 1-5 ranged from 2.6 Gy to 3.1 Gy and 1.9 Gy to 2.3 Gy for $\mathrm{HDR}_{\text {PointA }}$ and $\mathrm{HDR}_{\mathrm{VOL}}$ plans, respectively (Table 5). The median $\mathrm{D}_{2.0(\mathrm{HDR})}$ of sigmoid for fractions 1-5 ranged from 3.1 Gy to 3.3 Gy and 2.5 Gy to 2.7 Gy for HDR PointA and $\mathrm{HDR}_{\mathrm{VOL}}$ plans, respectively. The median bladder $\mathrm{D}_{2.0(\mathrm{HDR})}$ for fractions 1-5 ranged from 3.6 Gy to $4.3 \mathrm{~Gy}$ and 3.1 Gy to 3.2 Gy for HDR PointA $_{\text {and } H_{D D R}}$ plans, respectively (Table 5).
Table 3. High-dose-rate brachytherapy characteristics

\begin{tabular}{lc} 
HDR-BT parameters & $N=48$ \\
\hline HDR-BT applicators by fraction (fx) & \\
\hline T\&R $\times 5 \mathrm{fx}$ & $45(94 \%)$ \\
\hline T\&R $\times 4 \mathrm{fx}$ and T\&O $\times 1 \mathrm{fx}$ & $1(2 \%)$ \\
\hline T\&O $\times 5 \mathrm{fx}$ & $1(2 \%)$ \\
\hline T\&O $\times 3$ fx and T\&R $\times 2 \mathrm{fx}$ & $23(48 \%)$ \\
\hline Fractionation schemes & $25(52 \%)$ \\
\hline 5.5 Gy $\times 5$ fx & $34(71 \%)$ \\
\hline Others & $14(29 \%)$ \\
\hline Point A planning strategies: & \\
\hline Point A (2.0 cm) $\times 5 \mathrm{fx}$ & $23(18,33)$ \\
\hline Modified Point A or Point A & $52(35,63)$ \\
\hline Treatment volumes (cc), median (IQR): & $44(33,64)$ \\
\hline HR-CTV & $100(88,118)$ \\
\hline Rectum & $17(14,22)$ \\
\hline Sigmoid & \\
\hline Bladder & \\
\hline Duration (days), median (IQR): & \\
\hline
\end{tabular}

HDR-BT - high-dose-rate brachytherapy, N-total number of patients, T\&R - tandem and ring, T\&O -tandem and ovoids, HR-CTV-high-risk clinical target volume

The relative difference between the $\mathrm{D}_{90(\mathrm{EBRT}+\mathrm{HDR})}$ of the HR-CTV for $\mathrm{HDR}_{\text {PointA }}(98 \mathrm{~Gy})$ and $\mathrm{HDR}_{\mathrm{VOL}}$ (86 Gy) plans was $11 \%$ (Table 6). The hotspots within $\mathrm{HR}-\mathrm{CTV}$ were decreased by $\mathrm{HDR}_{\mathrm{VOL}}$-based planning for fractions $1-5$, with a relative difference of $9-24 \%$ for $\mathrm{V}_{150}$ and $10-30 \%$ for $\mathrm{V}_{200}$ compared to $\mathrm{HDR}_{\text {Point }}$ plans. The median HR-CTV $V_{150}$ for fractions 1-5 ranged from $72 \%$ to $79 \%$ and $62 \%$ to $65 \%$ for HDR PointA $_{\text {and HDR }}$ VOL plans, respectively (Table 6). The median HR-CTV $V_{200}$ for fractions 1-5 ranged from $46 \%$ to $55 \%$ and $39 \%$ to $41 \%$ for $\mathrm{HDR}_{\text {PointA }}$ and $\mathrm{HDR}_{\mathrm{VOL}}$ plans, respectively (Table 6). The conformal indexes of HR-CTV for HDR-BT fractions 1-5 were increased by $\mathrm{HDR}_{\mathrm{VOL}}$-based planning with a relative difference of $18-31 \%$ compared to HDR $_{\text {PointA }}$ plans and ranged from 0.29 to 0.35 and 0.44 to 0.48 for $\mathrm{HDR}_{\text {PointA }}$ and $\mathrm{HDR}_{\mathrm{VOL}}$ plans, respectively (Table 7).

Table 4. Gastrointestinal and genitourinary toxicity profile

\begin{tabular}{lcc} 
Status & $\begin{array}{c}\text { Grade 3-4 GI } \\
\text { toxicities }\end{array}$ & $\begin{array}{c}\text { Grade 3-4 GU } \\
\text { toxicities }\end{array}$ \\
\hline Present & $8(17 \%)$ & $7(15 \%)$ \\
\hline Absent & $35(73 \%)$ & $36(75 \%)$ \\
\hline Data missing & $5(10 \%)$ & $5(10 \%)$
\end{tabular}

Gl - gastrointestinal, GU - genitourinary 


\section{Discussion}

The primary objective of HDR-BT in cervical cancer is to provide a biologically significant radiation dose to the HR-CTV without compromising OAR. Current literature

Table 5. Dosimetric parameters of organs at risk

\begin{tabular}{|c|c|c|c|}
\hline $\begin{array}{l}\text { OAR } \\
\text { parameters }\end{array}$ & $\begin{array}{l}\text { HDR }_{\text {pointA }} \text { (Gy) } \\
\text { Median (IQR) }\end{array}$ & $\begin{array}{c}\mathrm{HDR}_{\mathrm{VOL}}(\mathrm{Gy}) \\
\text { Median (IQR) }\end{array}$ & $\begin{array}{c}\text { Relative } \\
\text { difference (\%) } \\
\text { Median (IQR) }\end{array}$ \\
\hline \multicolumn{4}{|l|}{ Rectum } \\
\hline $\mathrm{D}_{0.1(\mathrm{EBRT}+\mathrm{HDR})}$ & $73(68,80)$ & $63(57,70)$ & $10(5,22)$ \\
\hline $\mathrm{D}_{1.0(\mathrm{EBRT}+\mathrm{HDR})}$ & $66(62,69)$ & $58(53,63)$ & $9(4,17)$ \\
\hline $\mathrm{D}_{2.0(\mathrm{EBRT}+\mathrm{HDR})}$ & $63(59,66)$ & $56(52,60)$ & $8(3,15)$ \\
\hline \multicolumn{4}{|l|}{$\mathrm{D}_{2.0(\mathrm{HDR})}$} \\
\hline Fraction 1 & $2.8(3,4)$ & $2.3(3,3)$ & $19(2,57)$ \\
\hline Fraction 2 & $2.9(2,3)$ & $2.3(2,3)$ & $23(2,65)$ \\
\hline Fraction 3 & $3.1(2,4)$ & $1.8(2,3)$ & $34(8,91)$ \\
\hline Fraction 4 & $2.6(8,3)$ & $1.9(8,2)$ & $37(8,76)$ \\
\hline Fraction 5 & $2.6(8,3)$ & $1.9(8,3)$ & $31(11,86)$ \\
\hline
\end{tabular}

\begin{tabular}{llll}
\hline Sigmoid & & & \\
\hline $\mathrm{D}_{\text {0.1(EBRT+HDR) }}$ & $76(69,91)$ & $70(61,77)$ & $9(4,19)$ \\
\hline $\mathrm{D}_{1.0(\text { (EBRT+HDR) }}$ & $68(62,76)$ & $62(57,67)$ & $7(4,14)$ \\
\hline $\mathrm{D}_{2.0(\text { (EBRT+HDR) }}$ & $65(59,70)$ & $59(56,64)$ & $6(3,14)$ \\
\hline $\mathrm{D}_{2.0(\text { HDR })}$ & & & \\
\hline Fraction 1 & $3.3(3,4)$ & $2.7(3,3)$ & $10(1,51)$ \\
\hline Fraction 2 & $3.2(1,4)$ & $2.7(1,3)$ & $14(2,48)$ \\
\hline Fraction 3 & $3.3(2,4)$ & $2.6(2,3)$ & $19(5,49)$ \\
\hline Fraction 4 & $3.3(5,4)$ & $2.5(5,3)$ & $26(6,49)$ \\
\hline Fraction 5 & $3.1(6,4)$ & $2.6(6,3)$ & $25(10,45)$
\end{tabular}

\begin{tabular}{llll}
\hline Bladder & & & \\
\hline $\mathrm{D}_{0.1(\mathrm{EBRT}+\mathrm{HDR})}$ & $91(74,103)$ & $78(69,90)$ & $12(5,24)$ \\
\hline $\mathrm{D}_{1.0 \text { (EBRT+HDR) }}$ & $79(68,86)$ & $68(62,75)$ & $10(5,18)$ \\
\hline $\mathrm{D}_{2.0(\mathrm{EBRT}+\mathrm{HDR})}$ & $74(66,80)$ & $66(59,71)$ & $10(4,16)$ \\
\hline $\mathrm{D}_{2.0(\text { HDR })}$ & & & \\
\hline Fraction 1 & $3.8(4,5)$ & $3.1(4,4)$ & $8(1,45)$ \\
\hline Fraction 2 & $4.3(1,5)$ & $3.1(1,4)$ & $15(2,52)$ \\
\hline Fraction 3 & $4.1(2,5)$ & $3.1(2,4)$ & $21(4,53)$ \\
\hline Fraction 4 & $3.6(4,5)$ & $3.2(4,4)$ & $23(7,45)$ \\
\hline Fraction 5 & $4.0(7,5)$ & $3.2(7,4)$ & $22(9,45)$
\end{tabular}

OAR - organs at risk, HDR PointA - conventional Point A or modified Point A plan, $H D R_{V O L}$ - image-guided adaptive volume-based HDR-BT planning, $D_{O 1 \text { IEBRT+HDR }}$ - total $E Q D_{2}$ dose to $0.1 \mathrm{cc}$ from the combination of EBRT and HDR-BT, $D_{1 . O(E B R T+H D R)}$ - total $E Q D_{2}$ dose to $1.0 \mathrm{cc}$ from the combination of $E B R T$ and $H D R-B T ; D_{2} O(E B R T+H D R)$ - total EQD2 dose to $2.0 \mathrm{cc}$ from the combination of EBRT and HDR-BT; $D_{2.0(H D R)}$ - dose to $2.0 \mathrm{cc}$ per HDR-BT fraction attributes GI and GU toxicity from gynecological radiation to the high OAR point doses and recommend maintaining the total $\mathrm{EQD}_{2} \mathrm{D}_{2 \mathrm{cc}}$ of the rectum, sigmoid, and bladder to be $<70 \mathrm{~Gy},<70-75 \mathrm{~Gy}$, and $<90 \mathrm{~Gy}$, respectively $[1,2,23,31,32,33]$. Despite the Point A plans in our study, meeting the recommended OAR dose constraints, we observed $15 \%$ and $17 \%$ of patients having grade $3-4$ GU and GI toxicities, respectively. The significance of OAR dose limits per HDR-BT fraction in predicting GI and GU toxicities is an unexplored arena with no clear consensus on the OAR dose limits per HDR-BT fraction. Our study for the first time reports the range of $\mathrm{D}_{2 \mathrm{cc}}$ of rectum, sigmoid, and bladder per HDR-BT fraction for Point A plans and its improvement by $\mathrm{HDR}_{\mathrm{VOL}}$ planning. Our

Table 6. Dosimetric parameters of high-risk clinical target volume

\begin{tabular}{|c|c|c|c|}
\hline $\begin{array}{l}\text { HR-CTV } \\
\text { parameters }\end{array}$ & $\begin{array}{l}\text { HDR }_{\text {PointA }} \\
\text { Median } \\
(\mathrm{IQR}) \\
\end{array}$ & $\begin{array}{l}\mathrm{HDR}_{\text {VoL }} \\
\text { Median } \\
\text { (IQR) }\end{array}$ & $\begin{array}{c}\text { Relative } \\
\text { difference (\%) } \\
\text { Median (IQR) }\end{array}$ \\
\hline \multicolumn{4}{|l|}{$\mathrm{V}_{150(\mathrm{HDR})}(\%)$} \\
\hline Fraction 1 & $73(62,88)$ & $62(55,67)$ & $9(2,39)$ \\
\hline Fraction 2 & $73(60,90)$ & $63(58,67)$ & $12(1,38)$ \\
\hline Fraction 3 & $72(64,89)$ & $62(57,65)$ & $13(3,41)$ \\
\hline Fraction 4 & $79(64,89)$ & $65(59,68)$ & $20(3,37)$ \\
\hline Fraction 5 & $79(71,89)$ & $63(58,67)$ & $24(5,44)$ \\
\hline \multicolumn{4}{|l|}{$V_{200(\mathrm{HDR})}(\%)$} \\
\hline Fraction 1 & $47(39,63)$ & $39(33,45)$ & $10(1,53)$ \\
\hline Fraction 2 & $48(38,66)$ & $40(37,45)$ & $14(1,52)$ \\
\hline Fraction 3 & $46(40,58)$ & $40(35,43)$ & $15(0,51)$ \\
\hline Fraction 4 & $55(41,66)$ & $41(36,45)$ & $22(1,62)$ \\
\hline Fraction 5 & $54(44,60)$ & $41(36,44)$ & $30(1,53)$ \\
\hline $\mathrm{D}_{90 \text { (EBRT+HDR) }}$ (Gy) & $98(91,105)$ & $86(84,87)$ & $11(4,22)$ \\
\hline
\end{tabular}

$V_{150(H D R)}$ - percentage of HR-CTV receiving $150 \%$ of the prescription dose per HDR-BT fraction, $V_{200(H D R)}$ - percentage of HR-CTV receiving $200 \%$ of the prescription dose per HDR-BT fraction, $D_{90(E B R T+H D R)}$ - dose to $90 \%$ of the HR-CTV from the combination of EBRT and HDR-BT

Table 7. Conformal index per high-dose-rate brachytherapy fraction

\begin{tabular}{lccc}
$\begin{array}{l}\text { HDR-BT } \\
\text { fraction }\end{array}$ & $\begin{array}{c}\mathrm{HDR}_{\text {PointA }} \\
\text { Median (IQR) }\end{array}$ & $\begin{array}{c}\mathrm{HDR}_{\text {VOL }} \\
\text { Median (IQR) }\end{array}$ & $\begin{array}{c}\text { Relative dif- } \\
\text { ference (\%) } \\
\text { Median (IQR) }\end{array}$ \\
\hline 1 & $0.35(0.25,0.40)$ & $0.44(0.38,0.52)$ & $-18(-46,-1)$ \\
\hline 2 & $0.29(0.22,0.41)$ & $0.44(0.39,0.50)$ & $-28(-50,-2)$ \\
\hline 3 & $0.32(0.22,0.39)$ & $0.48(0.39,0.54)$ & $-29(-49,-10)$ \\
\hline 4 & $0.29(0.22,0.40)$ & $0.47(0.38,0.55)$ & $-29(-48,-6)$ \\
\hline 5 & $0.31(0.25,0.38)$ & $0.46(0.40,0.55)$ & $-31(-48,-12)$
\end{tabular}


institutional goals for $\mathrm{D}_{2 \mathrm{cc}}$ of the rectum, sigmoid, and bladder per HDR-BT fraction are approximately < 3.0-3.5 Gy, $<3.0-4.0 \mathrm{~Gy}$, and < 4.0-4.5 Gy, respectively. However, such an objective is often not met in clinical scenarios, where HR-CTV is either at sub-centimeter proximity or is flushing the OAR. Under such circumstances, physicians either exceed the OAR dose limits or apply a lower prescription dose on the respective HDR-BT fraction with objective of meeting the total $\mathrm{EQD}_{2}$ dose constraints. The clinical consequences of such scenarios in terms of GI and GU toxicities are unknown and require an understanding of the OAR dose constraints per HDR-BT fraction within the context of the OAR that are simultaneously recovering from approximately 35-45 Gy of EBRT and receiving the remaining $\mathrm{RT}$. In current era of IMRT, the incidence of grade 3-4 GU and GI toxicities have significantly decreased, compared to conventional AP/PA and four field box techniques [31,33]. 50\% of our patients were treated with 3DRT and $67 \%$ had a pelvic sidewall boost with AP/PA fields. Furthermore, $52 \%$ of our patients had nodal boost by either IMRT or 3DRT or both, which was not used to calculate $\mathrm{EQD}_{2}$ (EBRT + HDR-BT) doses to the OAR. Thus, the GI and GU toxicities we observed in our study population might have been due to a multitude of factors such as dose per HDR-BT fraction, treatment modality, and total dose.

The evolution of 3D planning from 2D planning resulted in the advent of volume-based HDR-BT planning, where the dose can be prescribed to the HR-CTV delineated by CT and/or MRI scans with close monitoring of OAR doses $[7,8]$. Accurate target and OAR delineation led to $\mathrm{T} \& \mathrm{R}$ and $\mathrm{T} \& \mathrm{O}$ based dose optimization techniques to create conformal plans by altering dwell times and dwell positions $[34,35,36]$. Several studies have shown the benefits of image-guided volume-based brachytherapy planning in gynecological malignancies compared to Point A-based plans [20,21,22,23,24,25]. Point A-based plans were compared with volume-based plans by Shin et al., and demonstrated significant improvement in OAR doses and conformal indexes by volume-based planning [21]. In the study by Onal et al., CT-guided volume-based plans showed significant improvement in $\mathrm{D}_{2 \mathrm{cc}}$ and $\mathrm{D}_{5 \mathrm{cc}}$ compared to traditional Point A-based ICRU bladder doses [20]. Similarly, our study demonstrates the advantages of volume-based HDR planning in improving the OAR doses and conformality. In the last decade, many academic institutions have led further advancements in volume-based HDR-BT planning for cervical cancer with the integration of MRI [22,23,24,25,31,32,37,38,39]. The ability of MRI in better defining HR-CTV dimensions and improving the doses to the HR-CTV was demonstrated by Viswanathan et al. and Tanderup et al. [24,25]. Studies by Pötter et al. and Lindegaard et al. showed better local control and reduction in toxicities by image-guided volume-based HDR planning compared to historical series $[22,23,40,41]$. However, most community practices do not have a dedicated MRI scanner available for brachytherapy planning due to financial and infrastructure challenges. Recent reports propose a hybrid CT/ MRI approach to circumvent this problem, where an MRI is used for the initial HDR-BT fraction followed by CT scans for the remaining fractions $[27,40,41]$. In our study, we used a similar approach where an MRI was obtained 1-2 weeks before the first HDR, which was fused with successive CT scans obtained for each HDR-BT fraction.

Estimating point doses on the walls of hollow structures such as rectum, sigmoid, and bladder are not always precise due to inter-fraction and intra-fraction movements as well as limitations of CT in soft tissue delineation [2]. In our study, we demonstrated improvements in $D_{0.1 c c}, D_{1 c c}$ and $D_{2 c c}$ to the rectum, sigmoid, and bladder by volume-based planning. We did not use $D_{5 c c}$ and $D_{10 \mathrm{cc}}$ because accurate estimation of $5 \mathrm{cc}$ and $10 \mathrm{cc}$ require precise contouring of the rectal, sigmoid, and bladder walls with MRI per HDR-BT fraction [2]. Georg et al. demonstrates $\mathrm{D}_{1 \mathrm{cc}}$ and $\mathrm{D}_{2 \mathrm{cc}}$ as significant parameters in predicting rectal toxicity, while $\mathrm{D}_{0.1 \mathrm{cc}}, \mathrm{D}_{1 \mathrm{cc}}$, and $\mathrm{D}_{2 \mathrm{cc}}$ were significant in predicting bladder toxicities [42]. $\mathrm{D}_{0.1 \mathrm{cc}}, \mathrm{D}_{1 \mathrm{cc}}, \mathrm{D}_{2 \mathrm{cc}}$ and $\mathrm{D}_{5 \mathrm{cc}}$ were shown to be predictive of grade 2 or more recto-sigmoidal mucosal changes by Koom et al., whereas Kim et al. reports $\mathrm{D}_{5 \mathrm{cc}}$ as predictive of rectosigmoid mucosal changes and late rectosigmoid complications $[43,44]$. Accurate evaluation of OAR point doses is of paramount importance to prevent GI and GU toxicities after brachytherapy. However, the calculation of EQD2 (EBRT + HDR-BT)-based point doses to OAR is based on the fundamental assumption of it being on the same anatomical spot during EBRT and brachytherapy despite organ and applicator movements. In addition, current $\mathrm{EQD}_{2}$ calculations do not account for hotspot locations from the EBRT portion of RT. Thus, our current methods carry the inherent disadvantage of inaccurate anatomical point dose assessments. In recent years, the role of deformable image registration (DIR) has become increasingly relevant in accurately estimating the anatomical point dose when doses from different scans are combined $[45,46,47,48]$. DIR is still an active area of investigation and further work in this field will help us accurately estimate OAR point doses when EBRT and BT doses are combined.

Twelve percent of the 240 plans re-planned by $\mathrm{HDR}_{\mathrm{VOL}}$ planning were rejected in our study due to inferior HR-CTV and OAR doses. $20 \%$ of the 240 plans reviewed used a modified Point A technique instead of the conventional Point A due to similar reasons. Radiologically, such patients had one or all the following characteristics: 1. parametrial extension; 2. pelvic sidewall extension; 3. OAR (rectum, sigmoid, or bladder) flushing at the surface of HR-CTV. Volume-based planning in such scenarios resulted in either under dosing of the HR-CTV or over-dosing of the OAR compared to Point A plans in our experience. Parametrial and pelvic sidewall tumor extensions impose challenges for HDR-BT planning using conventional CT compatible T\&R and T\&O. The challenge is in the dosimetric and geometric limitations of the traditional $\mathrm{T} \& \mathrm{R}$ and $\mathrm{T} \& \mathrm{O}$ applicators, where the only variables that can be changed are dwell times and dwell positions, which are restricted to the tandem, ovoid, and ring. Literature indicates limitations of volume-based planning in delivering adequate dose to asymmetrical tumors with diameter greater than $4 \mathrm{~cm}$ with conventional T\&R and T\&O applicators [21]. The study by Shin et al. demonstrated similar 
results, where volume-based planning led to an increase in rectum and bladder doses in patients where the HR-CTV was not covered by the $100 \%$ isodose line [21]. An alternative to the traditional T\&R and T\&O applicators is the Vienna applicator that has additional channels for needle insertions around the tandem. The Vienna applicator combines an intra-cavitary and interstitial approach and thus adds an additional variable through additional loading channels to attain desirable plans with clinically acceptable HR-CTV doses and lower OAR doses. The studies by Kirisitis et al. and Hsu et al. have demonstrated the potential of Vienna applicators in HDR-BT treatments of cervical cancer patients in obtaining lowered OAR doses without compromising HR-CTV doses $[49,50]$. Thus, Vienna applicators are a possible solution for HDR-BT planning in anatomically challenging clinical scenarios.

Point A corresponds to the para-cervical triangle with the uterine vessels and ureter crossing within, where the microscopic and macroscopic disease exist [7,8,11,12]. Historically, EQD 2 doses of 80-90 Gy to the high-risk disease defined by Point $\mathrm{A}$ were required to achieve local control rates of $80-90 \%$ for cervical cancer $[3,4,5,6]$. However, in recent years, several studies have demonstrated comparable local control rates with MRI-guided HDR-BT planning for cervical cancer without compromising the total $\mathrm{EQD}_{2}$ dose to the HR-CTV $[2,22,23,37]$. Our data clearly demonstrates the potential of $\mathrm{HDR}_{\mathrm{VOL}}$ planning in achieving the recommended $D_{90}$ of the HR-CTV with a median dose of $86 \mathrm{~Gy}$. In addition to $\mathrm{D}_{90}$, current guidelines also recommend documentation of $\mathrm{V}_{150}$ and $\mathrm{V}_{200}$ of HR-CTV [2]. Nevertheless, a biological correlation between HR-CTV hotspots and local control for cervical cancer is unknown. In our study, we could achieve median HR-CTV hotspots of greater than $62 \%\left(\mathrm{~V}_{150}\right)$ and 39\% $\left(\mathrm{V}_{200}\right)$ by volume-based planning and were comparable to the data reported by other studies $[51,52]$.

\section{Conclusions}

Volume-based HDR-BT planning allows the physician to create a conformal dose cloud with respect to the HR-CTV after careful CT/MRI-based assessment of the tumor and OAR. Thus, the likelihood of over-estimation and under-estimation of doses to the HR-CTV and OAR, respectively, is lowered. Overall, our study demonstrates the dosimetric benefits of $\mathrm{HDR}_{\mathrm{VOL}}$ planning in lowering OAR doses and without compromising the recommended $\mathrm{D}_{90}$ of 80-90 Gy to the HR-CTV. The study also underscores the need for prospective studies investigating the OAR dose constraints per HDR-BT fraction and more accurate point dose assessment using DIR.

\section{Acknowledgements}

We would like to thank Dr. Michael C. Joiner for his valuable guidance during the design phase of the project.

\section{Disclosure}

The authors report no conflict of interest.

\section{References}

1. Viswanathan AN, Beriwal S, De Los Santos JF et al. American Brachytherapy Society consensus guidelines for locally advanced carcinoma of the cervix. Part II: high-dose-rate brachytherapy. Brachytherapy 2012; 11: 47-52.

2. Pötter R, Haie-Meder C, Van Limbergen E et al. Recommendations from gynaecological (GYN) GEC ESTRO working group (II): concepts and terms in 3D image-based treatment planning in cervix cancer brachytherapy-3D dose volume parameters and aspects of 3D image-based anatomy, radiation physics, radiobiology. Radiother Oncol 2006; 78: 67-77.

3. Fletcher GH, Shukovsky LJ. The interplay of radiocurability and tolerance in the irradiation of human cancers. J Radiol Electrol Med Nucl 1975; 56: 383-400.

4. Dimopoulos JC, Lang S, Kirisits C et al. Dose-volume histogram parameters and local tumor control in magnetic resonance image-guided cervical cancer brachytherapy. Int J Radiat Oncol Biol Phys 2009; 75: 56-63.

5. Charra-Brunaud C, Harter V, Delannes $M$ et al. Impact of 3D image-based PDR brachytherapy on outcome of patients treated for cervix carcinoma in France: results of the French STIC prospective study. Radiother Oncol 2012; 103: 305-313.

6. Pötter R, Knocke TH, Fellner C et al. Definitive radiotherapy based on HDR brachytherapy with iridium 192 in uterine cervix carcinoma: report on the Vienna University Hospital findings (1993-1997) compared to the preceding period in the context of ICRU 38 recommendations. Cancer Radiother 2000; 4: 159-172.

7. Srivastava A, Datta NR. Brachytherapy in cancer cervix: Time to move ahead from point A? World J Clin Oncol 2014; 5: 764-774.

8. Vargo JA, Beriwal S. Image-based brachytherapy for cervical cancer. World J Clin Oncol 2014; 5: 921-930.

9. Eifel PJ, Ho A, Khalid N et al. Patterns of radiation therapy practice for patients treated for intact cervical cancer in 2005 to 2007: a quality research in radiation oncology study. Int J Radiat Oncol Biol Phys 2014; 89: 249-256.

10. Narayan K, van Dyk S, Bernshaw D et al. Comparative study of LDR (Manchester system) and HDR image-guided conformal brachytherapy of cervical cancer: patterns of failure, late complications, and survival. Int J Radiat Oncol Biol Phys 2009; 74: 1529-1535.

11. Tod M, Meredith WJ. Treatment of cancer of the cervix uteri, a revised Manchester method. Br J Radiol 1953; 26: 252-257.

12. Tod MC, Tod MC, Meredith WJ. A Dosage System for Use in the Treatment of Cancer of the Uterine Cervix. Br J Radiol 1938; 11: 809-824.

13. Datta NR, Kumar S, Das KJ et al. Variations of intracavitary applicator geometry during multiple HDR brachytherapy insertions in carcinoma cervix and its influence on reporting as per ICRU report 38. Radiother Oncol 2001; 60: 15-24.

14. Katz A, Eifel PJ. Quantification of intracavitary brachytherapy parameters and correlation with outcome in patients with carcinoma of the cervix. Int J Radiat Oncol Biol Phys 2000; 48: 1417-1425.

15. Wang KL, Yang YC, Chao KS et al. Correlation of traditional point a with anatomic location of uterine artery and ureter in cancer of the uterine cervix. Int J Radiat Oncol Biol Phys 2007; 69: 498-503.

16. Datta NR, Srivastava A, Maria Das KJ et al. Comparative assessment of doses to tumor, rectum, and bladder as evaluated by orthogonal radiographs vs. computer enhanced computed tomography-based intracavitary brachytherapy in cervical cancer. Brachytherapy 2006; 5: 223-229.

17. Kim H, Beriwal S, Houser C et al. Dosimetric analysis of 3D image-guided HDR brachytherapy planning for the treat- 
ment of cervical cancer: is point A-based dose prescription still valid in image-guided brachytherapy? Med Dosim 2011; 36: 166-170.

18. Kim RY, Pareek P. Radiography-based treatment planning compared with computed tomography (CT)-based treatment planning for intracavitary brachytherapy in cancer of the cervix: analysis of dose-volume histograms. Brachytherapy 2003; 2: $200-206$

19. Kim RY, Shen S, Duan J. Image-based three-dimensional treatment planning of intracavitary brachytherapy for cancer of the cervix: dose-volume histograms of the bladder, rectum, sigmoid colon, and small bowel. Brachytherapy 2007; 6: 187-194.

20. Onal C, Arslan G, Topkan E et al. Comparison of conventional and CT-based planning for intracavitary brachytherapy for cervical cancer: target volume coverage and organs at risk doses. J Exp Clin Cancer Res 2009; 28: 95.

21. Shin $\mathrm{KH}, \mathrm{Kim} \mathrm{TH}, \mathrm{Cho} \mathrm{JK}$ et al. CT-guided intracavitary radiotherapy for cervical cancer: Comparison of conventional point A plan with clinical target volume-based three-dimensional plan using dose-volume parameters. Int J Radiat Oncol Biol Phys 2006; 64: 197-204.

22. Lindegaard JC, Fokdal LU, Nielsen SK et al. MRI-guided adaptive radiotherapy in locally advanced cervical cancer from a Nordic perspective. Acta Oncol 2013; 52: 1510-1519.

23. Pötter R, Georg P, Dimopoulos JC et al. Clinical outcome of protocol based image (MRI) guided adaptive brachytherapy combined with 3D conformal radiotherapy with or without chemotherapy in patients with locally advanced cervical cancer. Radiother Oncol 2011; 100: 116-123.

24. Tanderup K, Nielsen SK, Nyvang GB et al. From point A to the sculpted pear: MR image guidance significantly improves tumour dose and sparing of organs at risk in brachytherapy of cervical cancer. Radiother Oncol 2010; 94: 173-180.

25. Viswanathan AN, Dimopoulos J, Kirisits C et al. Computed tomography versus magnetic resonance imaging-based contouring in cervical cancer brachytherapy: results of a prospective trial and preliminary guidelines for standardized contours. Int J Radiat Oncol Biol Phys 2007; 68: 491-498.

26. Kim HS, Song YS. International Federation of Gynecology and Obstetrics (FIGO) staging system revised: what should be considered critically for gynecologic cancer? J Gynecol Oncol 2009; 20: 135-136.

27. Beriwal S, Kannan N, Kim H et al. Three-dimensional high dose rate intracavitary image-guided brachytherapy for the treatment of cervical cancer using a hybrid magnetic resonance imaging/computed tomography approach: feasibility and early results. Clin Oncol (R Coll Radiol) 2011; 23: 685-690.

28. Lee LJ, Sadow CA, Russell A et al. Correlation of point B and lymph node dose in 3D-planned high-dose-rate cervical cancer brachytherapy. Int J Radiat Oncol Biol Phys 2009; 75: 803-809.

29. Haie-Meder C, Pötter R, Van Limbergen E et al. Recommendations from Gynaecological (GYN) GEC-ESTRO Working Group (I): concepts and terms in 3D image based 3D treatment planning in cervix cancer brachytherapy with emphasis on MRI assessment of GTV and CTV. Radiother Oncol 2005; 74: 235-245.

30. Nag S, Erickson B, Thomadsen B et al. The American Brachytherapy Society recommendations for high-dose-rate brachytherapy for carcinoma of the cervix. Int I Radiat Oncol Biol Phys 2000; 48: 201-211.

31. Gandhi AK, Sharma DN, Rath GK et al. Early clinical outcomes and toxicity of intensity modulated versus conventional pelvic radiation therapy for locally advanced cervix carcinoma: a prospective randomized study. Int J Radiat Oncol Biol Phys 2013; 87: 542-548.
32. Kang HC, Shin KH, Park SY et al. 3D CT-based high-doserate brachytherapy for cervical cancer: clinical impact on late rectal bleeding and local control. Radiother Oncol 2010; 97: 507-513.

33. Viswanathan AN, Lee LJ, Eswara JR et al. Complications of pelvic radiation in patients treated for gynecologic malignancies. Cancer 2014; 120: 3870-3883.

34. Chakrabarti B, Basu-Roy S, Kar SK et al. Comparison of dose volume parameters evaluated using three forward planning - optimization techniques in cervical cancer brachytherapy involving two applicators. J Contemp Brachytherapy 2017; 9: 431-445.

35. Dumane VA, Yuan Y, Sheu RD et al. Computed tomography-based treatment planning for high-dose-rate brachytherapy using the tandem and ring applicator: influence of applicator choice on organ dose and inter-fraction adaptive planning. J Contemp Brachytherapy 2017; 9: 279-286.

36. Rangarajan R. Dosimetric evaluation of image based brachytherapy using tandem ovoid and tandem ring applicators. Rep Pract Oncol Radiother 2018; 23: 57-60.

37. Gill BS, Kim H, Houser CJ et al. MRI-guided high-dose-rate intracavitary brachytherapy for treatment of cervical cancer: the University of Pittsburgh experience. Int J Radiat Oncol Biol Phys 2015; 91: 540-547.

38. Lim K, Small W Jr., Portelance L et al. Consensus guidelines for delineation of clinical target volume for intensity-modulated pelvic radiotherapy for the definitive treatment of cervix cancer. Int J Radiat Oncol Biol Phys 2011; 79: 348-355.

39. Eskander RN, Scanderbeg D, Saenz CC et al. Comparison of computed tomography and magnetic resonance imaging in cervical cancer brachytherapy target and normal tissue contouring. Int J Gynecol Cancer 2010; 20: 47-53.

40. Nemoto MW, Iwai Y, Togasaki G et al. Preliminary results of a new workflow for MRI/CT-based image-guided brachytherapy in cervical carcinoma. Jpn J Radiol 2017; 35: 760765 .

41. Nesvacil N, Pötter R, Sturdza A et al. Adaptive image guided brachytherapy for cervical cancer: a combined MRI-/CT-planning technique with MRI only at first fraction. Radiother Oncol 2013; 107: 75-81.

42. Georg P, Lang S, Dimopoulos JC et al. Dose-volume histogram parameters and late side effects in magnetic resonance imageguided adaptive cervical cancer brachytherapy. Int J Radiat Oncol Biol Phys 2011; 79: 356-362.

43. Kim TH, Kim JY, Sohn DK et al. A prospective observational study with dose volume parameters predicting rectosigmoidoscopic findings and late rectosigmoid bleeding in patients with uterine cervical cancer treated by definitive radiotherapy. Radiat Oncol 2013; 8: 28.

44. Koom WS, Sohn DK, Kim JY et al. Computed tomography-based high-dose-rate intracavitary brachytherapy for uterine cervical cancer: preliminary demonstration of correlation between dose-volume parameters and rectal mucosal changes observed by flexible sigmoidoscopy. Int J Radiat Oncol Biol Phys 2007; 68: 1446-1454.

45. Flower E, Do V, Sykes J et al. Deformable image registration for cervical cancer brachytherapy dose accumulation: Organ at risk dose-volume histogram parameter reproducibility and anatomic position stability. Brachytherapy 2017; 16: 387-392.

46. Hayashi K, Isohashi F, Akino $Y$ et al. Estimation of the total rectal dose of radical external beam and intracavitary radiotherapy for uterine cervical cancer using the deformable image registration method. J Radiat Res 2015; 56: 546-552.

47. Jamema SV, Mahantshetty U, Andersen E et al. Uncertainties of deformable image registration for dose accumulation of high-dose regions in bladder and rectum in locally advanced cervical cancer. Brachytherapy 2015; 14: 953-962. 
48. Teo BK, Bonner Millar LP, Ding X et al. Assessment of cumulative external beam and intracavitary brachytherapy organ doses in gynecologic cancers using deformable dose summation. Radiother Oncol 2015; 115: 195-202.

49. Hsu IC, Speight J, Hai J et al. A comparison between tandem and ovoids and interstitial gynecologic template brachytherapy dosimetry using a hypothetical computer model. Int J Radiat Oncol Biol Phys 2002; 52: 538-543.

50. Kirisits C, Lang S, Dimopoulos J et al. The Vienna applicator for combined intracavitary and interstitial brachytherapy of cervical cancer: design, application, treatment planning, and dosimetric results. Int J Radiat Oncol Biol Phys 2006; 65: 624630.

51. Bansal AK, Semwal MK, Sharma DN et al. A patient-based dosimetric study of intracavitary and interstitial brachytherapy in advanced stage carcinoma of the cervix. J Appl Clin Med Phys 2014; 15: 4509.

52. Kannan RA, Gururajachar JM, Ponni A et al. Comparison of manual and inverse optimisation techniques in high dose rate intracavitary brachytherapy of cervical cancer: A dosimetric study. Rep Pract Oncol Radiother 2015; 20: 365-369. 